\title{
Angular momentum of light revisited: Spin-orbit interactions in free space
}

\author{
Konstantin Y. Bliokh*a ${ }^{* a}$ Miguel A. Alonso ${ }^{\text {b,c }}$, Elena A. Ostrovskaya ${ }^{\mathrm{d}}$ \\ a Applied Optics Group, School of Physics, National University of Ireland, Galway, Galway, Ireland; \\ ${ }^{\mathrm{b}}$ The Institute of Optics, University of Rochester, Rochester, New York, 14627, USA \\ ${ }^{c}$ Department of Applied Physics, Aalto University, PO Box 13500, FI-00076 Aalto, Finland \\ ${ }^{\mathrm{d}}$ ARC Centre of Excellence for Quantum-Atom Optics and Nonlinear Physics Centre, Research \\ School of Physics and Engineering, The Australian National University, Canberra, Australia \\ *k.bliokh@gmail.com
}

\begin{abstract}
We give an exact self-consistent operator description of the spin and orbital angular momenta, position, and spin-orbit interactions of nonparaxial light in free space. We apply the general theory to symmetric and asymmetric Bessel beams exhibiting spin- and orbital-dependent intensity profiles. The exact wave solutions are clearly interpreted in terms of the Berry phases, quantization of caustics, and Hall effects of light, which can be readily observed experimentally.
\end{abstract}

Keywords: Optical angular momentum, polarization, nonparaxial light, spin-orbit interaction, angular momentum conversion, spin Hall effect of light

\section{INTRODUCTION}

The problem of the identification of the spin and orbital parts of the angular momentum (AM) of an electromagnetic wave has a long history and has posed fundamental difficulties both in quantum electrodynamics and classical optics. ${ }^{1-5}$ It is known that the photon AM operator in the momentum (plane-wave) representation has the form: ${ }^{1}$

$$
\hat{\mathbf{J}}=-i\left(\mathbf{k} \times \partial_{\mathbf{k}}\right)+\hat{\mathbf{S}} \equiv \hat{\mathbf{L}}+\hat{\mathbf{S}},
$$

where the orbital part is $\hat{\mathbf{L}}=\hat{\mathbf{r}} \times \hat{\mathbf{p}}\left(\hat{\mathbf{p}}=\mathbf{k}, \hat{\mathbf{r}}=i \partial_{\mathbf{k}}\right.$, we use units $\left.\hbar=c=1\right)$ and $\hat{\mathbf{S}}$ is the spin-1 operator given by $3 \times 3$ matrices $\left(\hat{S}_{a}\right)_{i j}=-i \varepsilon_{a i j}\left(\varepsilon_{a i j}\right.$ is the Levi-Civita symbol) which act on the Cartesian components of the wave electric field. The textbooks note that "The separation of the total AM into orbital and spin parts has restricted physical meaning. ... States with definite values of orbital and spin AM do not satisfy the condition of transversality in the general case."1-3

In classical optics, the two parts of Eq. (1) can be unambiguously associated with the orbital AM (OAM) and spin AM (SAM) for paraxial light, where the eigenmodes of $\hat{L}_{z}$ and $\hat{S}_{z}$ are circularly polarized vortex beams with the corresponding quantum numbers $\ell=0, \pm 1, \pm 2, \ldots$ (topological charge of the vortex $e^{i \ell \phi}$ ) and $\sigma= \pm 1$ (helicity). ${ }^{6,7}$ However, for non-paraxial fields the identification of OAM and SAM meets serious difficulties. ${ }^{2-5}$ Calculations based on the division of the Poynting energy flow into spin and orbital parts ${ }^{4,8-10}$ show that the non-paraxial correction to the OAM is proportional to $\sigma$ rather than to $\ell$.,9 This resulted in the conclusion that "In the general non-paraxial case there is no simple separation into $\ell$-dependent orbital and $\sigma$-dependent spin component of AM". ${ }^{4}$

Here we re-examine the problem and give an exact self-consistent solution in terms of the fundamental photon operators. Our approach generalizes and unifies previously disjointed results: (i) non-canonical OAM and SAM operators obtained for the second-quantized fields, ${ }^{2,3}$ (ii) non-commutative photon position operator and Berry monopole field in momentum space, ${ }^{11-14}$ (iii) separation of the spin and orbital parts of classical energy flows. ${ }^{8,9}$ We show that the $\sigma$ dependent non-paraxial part of the OAM arises from Berry-phase terms describing the spin-orbit interaction (SOI) of light. A similar effect occurs dynamically upon spin-to-orbital AM conversion in focusing and scattering of polarized light. ${ }^{10,15-19}$ Other manifestations of the SOI are the spin $^{20-29}$ and orbital ${ }^{30-37}$ Hall effects of light (i.e., $\sigma-$ and $\ell$ dependent transverse shifts of the field center of gravity) which are described by our position operator and take place

Complex Light and Optical Forces V, edited by David L. Andrews, Enrique J. Galvez, Jesper Glückstad, Proc. of SPIE Vol. 7950, 795003 - (c) 2011 SPIE · CCC code: 0277-786X/11/\$18 · doi: 10.1117/12.873818 
even in free space. ${ }^{29,37}$ We apply the general theory to the Bessel beams where the fundamental operators manifest themselves in immediately observable $\sigma$ - and $\ell$-dependent intensity distributions.

\section{GENERAL THEORY}

We consider a monochromatic electromagnetic field in free space, characterized by its plane-wave electric-field spectrum $\tilde{\mathbf{E}}(\mathbf{k})$. The SOI of light originates from the transversality constraint, $\mathbf{k} \cdot \tilde{\mathbf{E}}=0$, which couples polarization to the wave vector and reduces the full $3 \mathrm{D}$ vector space of the electric field components to the $2 \mathrm{D}$ subspace of the components tangential to a sphere of directions in $\mathbf{k}$-space. The operators $\hat{\mathbf{L}}$ and $\hat{\mathbf{S}}$ do not keep this subspace invariant, i.e., their action on a transverse mode results in a non-zero longitudinal component. ${ }^{1-3}$ However, this subspace is invariant for the total AM operator $\hat{\mathbf{J}}$, and one can divide it into two parts consistent with the transversality condition:

$$
\hat{\mathbf{J}}=\hat{\mathbf{L}}^{\prime}+\hat{\mathbf{S}}^{\prime}, \hat{\mathbf{L}}^{\prime}=\hat{\mathbf{L}}-\mathbf{\kappa} \times(\boldsymbol{\kappa} \times \hat{\mathbf{S}}), \hat{\mathbf{S}}^{\prime}=\boldsymbol{\kappa}(\boldsymbol{\kappa} \cdot \hat{\mathbf{S}}),
$$

where $\mathbf{\kappa}=\mathbf{k} / k$ and the modified OAM and SAM operators $\hat{\mathbf{L}}^{\prime}$ and $\hat{\mathbf{S}}^{\prime}$ can be regarded as projections of the operators $\hat{\mathbf{L}}$ and $\hat{\mathbf{S}}$ onto the transversality subspace., ${ }^{2,3}$

The modified SAM operator $\hat{\mathbf{S}}^{\prime}$ is proportional to the helicity operator $\hat{\sigma}=\boldsymbol{\kappa} \cdot \hat{\mathbf{S}}$, whereas the OAM operator can be written as $\hat{\mathbf{L}}^{\prime}=\hat{\mathbf{r}}^{\prime} \times \mathbf{k}$ with

$$
\hat{\mathbf{r}}^{\prime}=\hat{\mathbf{r}}+\frac{\mathbf{k} \times \hat{\mathbf{S}}}{k^{2}}=i \partial_{\mathbf{k}}+\frac{\mathbf{k} \times \hat{\mathbf{S}}}{k^{2}} .
$$

The modified position operator (3) has been considered in the context of photon localization and Berry phase. ${ }^{11-14,38,39}$ It describes the observable center of gravity of the field and brings about the space non-commutativity with the monopole term in $\mathbf{k}$-space:

$$
\left[\hat{r}_{i}^{\prime}, \hat{r}_{j}^{\prime}\right]=-i \varepsilon_{i j k} \hat{\sigma} \frac{k_{k}}{k^{3}}
$$

The operators $\hat{\mathbf{L}}^{\prime}$ and $\hat{\mathbf{S}}^{\prime}$ do not satisfy the AM algebra and have unusual commutation relations:

$$
\left[\hat{S}_{i}^{\prime}, \hat{S}_{j}^{\prime}\right]=0,\left[\hat{L}_{i}^{\prime}, \hat{L}_{j}^{\prime}\right]=i \varepsilon_{i j k} \hat{J}_{k}^{\prime},\left[\hat{L}_{i}^{\prime}, \hat{S}_{j}^{\prime}\right]=i \varepsilon_{i j k} \hat{S}_{k}^{\prime} .
$$

At the same time, the modified operators transform as vectors under rotations: $\left[\hat{J}_{i}, \hat{O}_{j}^{\prime}\right]=i \varepsilon_{i j l} \hat{O}_{l}^{\prime}, \hat{\mathbf{O}}^{\prime}=\hat{\mathbf{L}}^{\prime}, \hat{\mathbf{S}}^{\prime}$, and $\hat{\mathbf{r}}^{\prime}$.

The commutation relations (5) unveil similarity of operators $\hat{\mathbf{L}}^{\prime}$ and $\hat{\mathbf{S}}^{\prime}$ to those obtained for the second-quantized fields in Refs. 2 and 3. They are not AM operators and do not generate rotations, but it is suggested that they do correspond to observable continuous values of the OAM and SAM of non-paraxial transverse field. ${ }^{2,3}$

Remarkably, in the helicity representation the matrix components of the operators (2) and (3) become diagonal. We introduce spherical coordinates $(\theta, \phi, k)$ with basic vectors $\left(\mathbf{e}_{\theta}, \mathbf{e}_{\phi}, \mathbf{\kappa}\right)$ in $\mathbf{k}$-space, so that the free electric field has only $\left(\mathbf{e}_{\theta}, \mathbf{e}_{\phi}\right)$-components. The helicity basis of circular polarizations corresponds to the basic vectors $\mathbf{e}^{ \pm}=e^{ \pm i m \phi}\left(\mathbf{e}_{1} \pm i \mathbf{e}_{2}\right) / \sqrt{2}$, where $e^{ \pm i m \phi}$ is an arbitrary gauge factor. ${ }^{39}$ Transition from the global Cartesian field components $\left(\tilde{E}_{x}, \tilde{E}_{y}, \tilde{E}_{z}\right)^{T}$ to the helicity amplitudes $\left(\tilde{E}^{+}, \tilde{E}^{-}, \tilde{E}_{\|}\right)^{T}$ is realized via the local unitary transformation $\hat{U}(\theta, \phi)=\hat{R}_{z}(-\phi) \hat{R}_{y}(-\theta) \hat{R}_{z}(m \phi) \hat{V}$, where $\hat{R}_{a}(\alpha)=e^{i \alpha \hat{S}_{a}}$ is the matrix of rotation by an angle $\alpha$ with respect to the $a-$ axis, whereas $\hat{V}$ is the constant transformation from linear- to circular-polarization basis. Making the transformation of operators (2) and (3) to the helicity basis, $\hat{\mathbf{O}}^{\prime} \rightarrow \hat{U}^{\dagger} \hat{\mathbf{O}}^{\prime} \hat{U}$, we obtain:

$$
\hat{\mathbf{S}}^{\prime}=\mathbf{\kappa} \hat{\sigma}, \quad \hat{\mathbf{L}}^{\prime}=-i \mathbf{k} \times \partial_{\mathbf{k}}-\hat{\mathbf{A}}_{B} \times \mathbf{k}, \quad \hat{\mathbf{r}}^{\prime}=i \partial_{\mathbf{k}}-\hat{\mathbf{A}}_{B} .
$$


Here, the helicity is diagonal: $\hat{\sigma}=\operatorname{diag}(1,-1,0)$, and

$$
\hat{\mathbf{A}}_{B}=-\frac{\mathbf{k} \times \hat{\mathbf{S}}}{k^{2}}-i \hat{U}^{\dagger} \partial_{\mathbf{k}} \hat{U}=\frac{m-\cos \theta}{k \sin \theta} \hat{\sigma} \mathbf{e}_{\phi}
$$

is the Berry gauge field (connection) which corresponds to the monopole curvature $\hat{\mathbf{F}}_{B}=\partial_{\mathbf{k}} \times \hat{\mathbf{A}}_{B}=\hat{\sigma} \mathbf{k} / k^{3}$. ${ }^{12-14,38}$ Hereafter we choose the gauge $m=1$, which corresponds to the absence of the phase singularity (Dirac string) along the positive $z$-axis in Eq. (7), ${ }^{39}$ allowing a smooth transition to the paraxial case, $\theta \rightarrow 0$.

The measurable expectation values of the OAM, SAM, and coordinate obtained from the diagonal operators (6) and (7) take the form:

$$
\begin{gathered}
\mathbf{S}=\sigma\left\langle\tilde{E}^{\sigma}|\mathbf{\kappa}| \tilde{E}^{\sigma}\right\rangle, \mathbf{L}=\left\langle\tilde{E}^{\sigma}|\hat{\mathbf{L}}| \tilde{E}^{\sigma}\right\rangle-\sigma\left\langle\tilde{E}^{\sigma}\left|\mathbf{A}_{B} \times \mathbf{k}\right| \tilde{E}^{\sigma}\right\rangle, \\
\mathbf{r}=\left\langle\tilde{E}^{\sigma}\left|i \partial_{\mathbf{k}}\right| \tilde{E}^{\sigma}\right\rangle-\sigma\left\langle\tilde{E}^{\sigma}\left|\mathbf{A}_{B}\right| \tilde{E}^{\sigma}\right\rangle .
\end{gathered}
$$

Here $\mathbf{A}_{B}=\mathbf{e}_{\phi} k^{-1}(1-\cos \theta) / \sin \theta$, convolution implies summation over $\sigma= \pm 1$ and integration over the 2D sphere of directions in the $\mathbf{k}$-space, and we assume normalization $\left\langle\tilde{E}^{\sigma} \mid \tilde{E}^{\sigma}\right\rangle=1$. While the SAM is purely intrinsic (originindependent), the OAM, in general, has both intrinsic and extrinsic contributions: ${ }^{40,41}$

$$
\mathbf{L}^{\mathrm{ext}}=\mathbf{r} \times \mathbf{p}, \mathbf{L}^{\mathrm{int}}=\mathbf{L}-\mathbf{L}^{\mathrm{ext}},
$$

where $\mathbf{p}=\left\langle\tilde{E}^{\sigma}|\mathbf{k}| \tilde{E}^{\sigma}\right\rangle$ is the expectation value of the linear momentum.

Equations (8)-(10) contain all the main observable results related to the AM and SOI of light. First, the $\sigma$-dependent non-paraxial Berry-phase term in $\mathbf{L}$ should be associated with the spin-to-orbit AM conversion. ${ }^{9,10,15-19}$ Particular cases of this term have appeared in Refs. 4, 9, and 10. Second, the orbital ${ }^{30-37}$ and $\operatorname{spin}^{20-29}$ Hall effects of light are described by the two terms in the position (9). Indeed, for symmetric vortex distributions, $\mathbf{r}=(0,0, z)$, after integration over $\phi$, but any asymmetry of the field distribution along, say, the $x$-axis immediately causes $\ell$ - and $\sigma$-dependent shift along the orthogonal $y$-axis, $y \neq 0$ together with $p_{x} \neq 0$ (see example below).

We emphasize that our results (6)-(10) are exact and no approximations were made. They are equivalent to application of the canonical operators $\hat{\mathbf{L}}, \hat{\mathbf{S}}$, and $\mathbf{r}$ to the laboratory-frame field components $\left(\tilde{E}_{x}, \tilde{E}_{y}, \tilde{E}_{z}\right)^{T}$ supplied with the transversality condition. The description in the $\mathbf{k}$-dependent helicity basis brings about the Berry-phase terms representing the Coriolis-type derivatives of the basic vectors: $\left(\hat{\mathbf{A}}_{B}\right)_{i j}=-i \mathbf{e}_{i}^{*} \cdot\left(\partial_{\mathbf{k}}\right) \mathbf{e}_{j} \delta_{i j}$, where $\left(\mathbf{e}_{1}, \mathbf{e}_{2}, \mathbf{e}_{3}\right) \equiv\left(\mathbf{e}^{+}, \mathbf{e}^{-}, \mathbf{\kappa}\right)$.

We have also found that the same OAM and SAM, Eq. (8), follow from the recently-suggested approach based on the separation of the spin and orbital parts in the classical Poynting flow ${ }^{8,9}$ (this will be published elsewhere).

\section{APPLICATION TO BESSEL BEAMS}

Importantly, our theory has a number of directly observable consequences. As the simplest example we take non-paraxial vector Bessel-beam solutions which are eigenmodes of $\hat{J}_{z}$ constructed from plane waves with well-defined helicity $\sigma \cdot{ }^{3,9,39}$ The angular spectrum of such beams is

$$
\tilde{\mathbf{E}}_{\ell}^{\sigma}=\mathbf{e}^{\sigma}(\theta, \phi) \tilde{E}_{\ell}^{\sigma}(\theta, \phi), \quad \tilde{E}_{\ell}^{\sigma}=A^{\sigma} \delta\left(\theta-\theta_{0}\right) e^{i \ell \phi},
$$

where $A^{\sigma}$ is a constant amplitude and $\theta_{0}$ is the polar angle of conical distribution of the $\mathbf{k}$-vectors, Fig. 1(a). For the $z$ components of OAM and SAM, Eqs. (8), of a superposition of $\sigma= \pm 1$ beams (11) we obtain: ${ }^{42}$ 


\section{(a)}

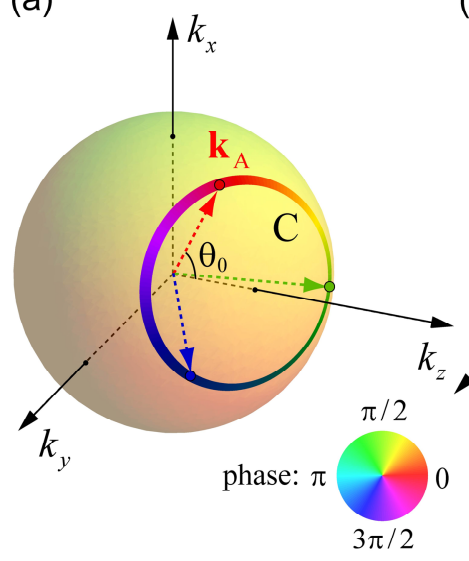

(b)

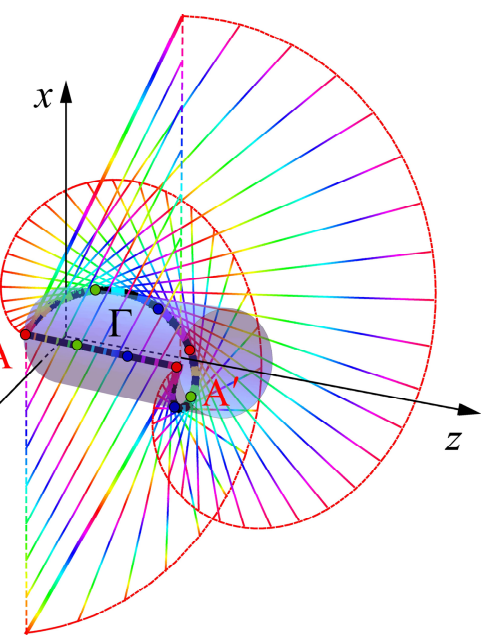

Figure 1. (Color online) (a) Bessel-beam distribution (11) on the sphere in $\mathbf{k}$-space with the azimuthal phase $2 \pi \ell$. (b) Cylindrical caustic in the real space, an example of the closed orbit $\Gamma$ on it, and the corresponding GO rays tangent to the caustic. Scalar phases are color-coded for $\ell=-1, \theta_{0}=\pi / 4$. Points A and A' on the caustic are connected by two paths: the straight line and the Poynting-flow helix. The phase matching yields the phase difference difference $2 \pi \ell$ between the paths and quantization of the caustic radius, Eq. (14). For circularlypolarized waves, the helical path brings about an additional Berry phase $\sigma \Phi_{B}$.

$$
L_{z}=\ell+\bar{\sigma} \frac{\Phi_{B}}{2 \pi}, S_{z}=\bar{\sigma}\left(1-\frac{\Phi_{B}}{2 \pi}\right), J_{z}=\ell+\bar{\sigma}
$$

where $\bar{\sigma}=\left(\left|A^{+}\right|^{2}-\left|A^{-}\right|^{2}\right) /\left(\left|A^{+}\right|^{2}+\left|A^{-}\right|^{2}\right)$ is the averaged helicity and $\Phi_{B}=\int_{C} \mathbf{A}_{B} \cdot d \mathbf{k}=2 \pi\left(1-\cos \theta_{0}\right)$ is the Berry phase associated with the contour $C=\left\{\theta=\theta_{0}, \phi \in(0,2 \pi)\right\}$ formed by the $\mathbf{k}$-vectors distribution on the sphere of directions, Fig. 1(a). ${ }^{43}$ Note that the $\bar{\sigma}$-dependent term in $L_{z}$ represents a "magnetic-monopole" flux contribution to the OAM, cf. Eq. (87) in Ref. 44. In the paraxial limit the Berry-phase term vanishes as $\Phi_{B} \approx \pi \theta_{0}^{2} \rightarrow 0$. The values (12) evidence an apparent partial conversion from SAM to OAM in non-paraxial light with the total AM being constant, ${ }^{4,9}$ akin to the spin-to-orbit AM conversion upon focusing of polarized light. ${ }^{9,10,15-19}$ Indeed, in the Richards-Wolf approximation, ${ }^{45}$ the focusing represents a geometric conical redirection of partial plane waves with their helicity being conserved. It is described exactly by the same transformation operator $\hat{U}(\theta, \phi)$ that describes transition to the helicity basis. ${ }^{19}$

Simultaneously with a $\sigma$-dependent OAM, the non-paraxial fields exhibit $\sigma$-dependent intensity distributions related to the modified position operator. The real-space intensity of the circularly-polarized Bessel beam, calculated via the Fourier transformation of Eq. (11), is

$$
I_{\ell}^{\sigma}(\xi) \propto\left|A_{\sigma}\right|^{2}\left[a^{2} J_{\ell}^{2}(\xi)+b^{2} J_{\ell+2 \sigma}^{2}(\xi)+2 a b J_{\ell+\sigma}^{2}(\xi)\right],
$$

where $(\rho, \varphi, z)$ are the cylindrical coordinates in real space, $a=\cos ^{2}\left(\theta_{0} / 2\right), b=\sin ^{2}\left(\theta_{0} / 2\right), \xi=k_{\perp} \rho, k_{\perp}=k \sin \theta_{0}$, and $J_{n}(\xi)$ are the Bessel functions of the first kind. The $\ell$ - and $\sigma$-dependence of the radial intensity profile (13) (Fig. 2) can be explained via a geometrical optics (GO) ray picture and the quantization of caustic underlying the maximum of the intensity. The rays associated with a Bessel beam are those that form an angle $\theta_{0}$ with the $z$-axis and touch a cylindrical caustic of radius $\rho=\rho_{\ell}^{\sigma},{ }^{46} \mathrm{Fig}$. 1. The quantization condition for a closed orbit $\Gamma$ is $\int_{\Gamma} \mathbf{k} \cdot d \mathbf{r}=2 \pi \ell$. Using the underlying position (6) $\mathbf{r}^{\prime \sigma}=\mathbf{r}-\sigma \mathbf{A}_{B}$, we observe that the Berry phase changes the effective optical length of 
a closed orbit on the cylindrical surface, Fig. 1(b). For the orbit $\Gamma=\left\{\rho=\rho_{\ell}^{\sigma}, \varphi \in(0,2 \pi)\right\}$ it becomes $k_{\perp}\left[2 \pi \operatorname{sgn}(\ell) \rho_{\ell}^{\sigma}-\sigma \Phi_{B}\right]$, which yields

$$
k_{\perp} \rho_{\ell}^{\sigma}=\left|\ell+\sigma \frac{\Phi_{B}}{2 \pi}\right| .
$$

Similar Berry-phase effects appear in quantum quantization problems, ${ }^{47,48}$ e.g., the half-integer Hall effect in graphene. ${ }^{49,50}$ Note also the exact correspondence between the GO caustic (14) and the wave OAM (12), $L_{z}=\left|k_{\perp} \rho_{\ell}^{\sigma}\right|$, which reflects the OAM interpretation as $\mathbf{r} \times \mathbf{k}$ for the rays. Comparison between the exact wave profiles (13) and the GO caustics (14) is shown in Fig. 2. The $\sigma$-dependent radial distribution in non-paraxial vortex fields can be measured experimentally by tightly focusing paraxial light with different polarizations, cf. Refs. 51 and 52.
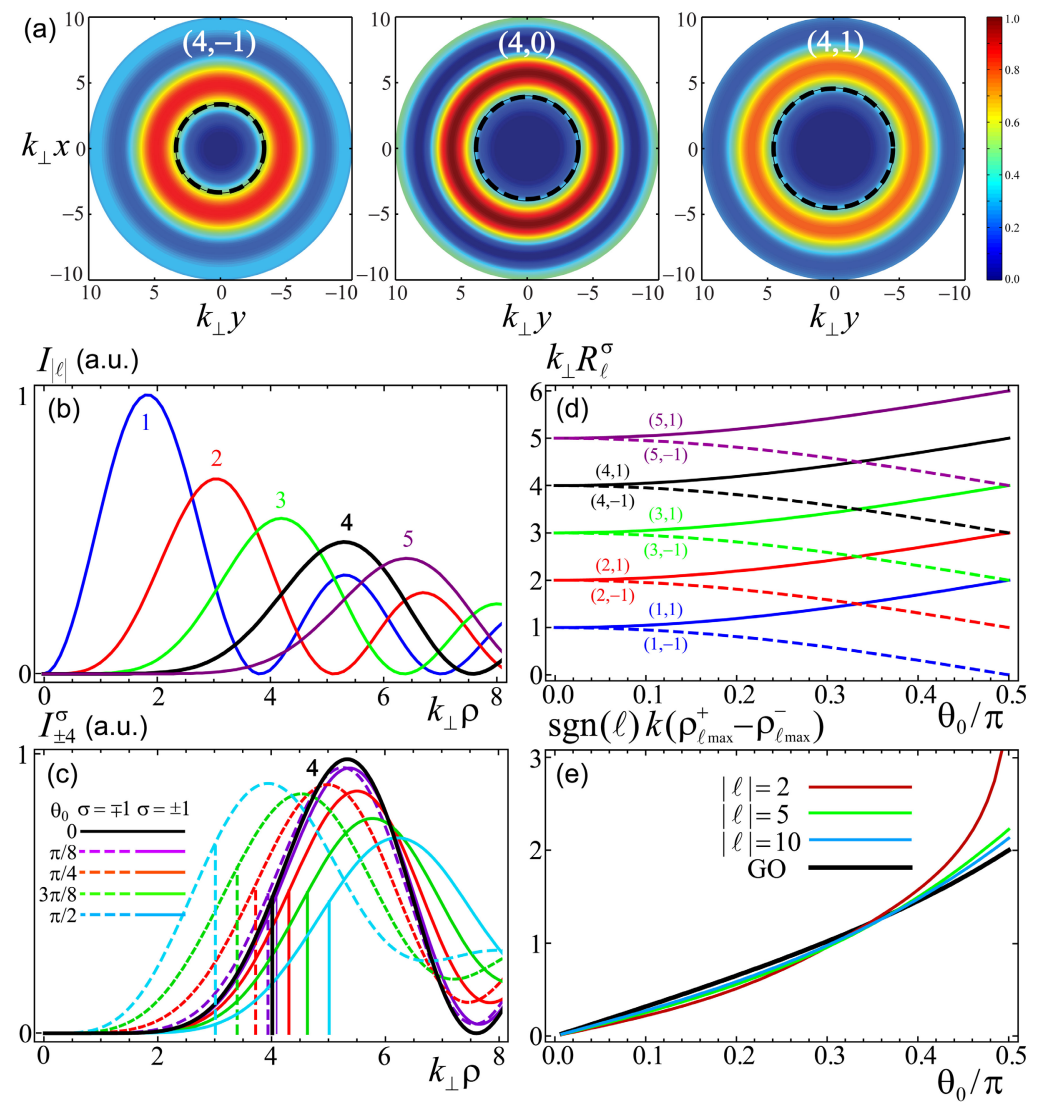

Figure 2. (Color online) (a) Intensity distributions, Eq. (13), marked by quantum numbers $(\ell, \sigma)$ for Bessel beams with $\theta_{0}=3 \pi / 8$. The spin-dependent profiles are shown for $\ell=4$ with $\sigma=-1,0$ (scalar case), and 1 . Dashed circles indicate the GO caustics (14). (b) Radial intensity profiles of the scalar $(\sigma=0)$ or paraxial $\left(\theta_{0} \rightarrow 0\right)$ Bessel beams $I_{\ell} \propto J_{\ell}^{2}(\xi)$. (c) The SOI splitting of the profile of the polarized non-paraxial Bessel beam $I_{ \pm 4}^{\sigma}(\xi)$, Eq. (13), at different values of $\theta_{0}$; vertical lines indicate GO caustics (14), cf. (a). (d) The GO caustics (14) marked by $\operatorname{sgn}(\ell)(\ell, \sigma)$ as functions of $\theta_{0}$. (e) The SOI splitting of the maxima of intensity (13) [cf. (c)] as dependent on $\theta_{0}$, approaching the GO limit (14) at $|\ell|>>1$. 
Finally, we consider the Hall effects of light described by the position (9). For this purpose we break the symmetry of the Bessel beams (11) along the $k_{x}$-axis and assume that the plane-wave components are distributed in the range $\phi \in(-\delta, \delta), 0<\delta<\pi$. (Such truncated azimuthal distributions can be generated via focusing by the corresponding sector of a lens. $\left.{ }^{26,28}\right)$ This yields a tilted beam with $p_{x}=\gamma k_{\perp}, \gamma=(\sin \delta) / \delta$ and a transversely shifted center of gravity (9):

$$
k_{\perp} y_{\ell}^{\sigma}=-\gamma\left(\ell+\bar{\sigma} \frac{\Phi_{B}}{2 \pi}\right)
$$

which closely resembles Eqs. (12) and (14). The $\ell$ - and $\sigma$-dependent parts of the shift (15) describe the orbital and spin Hall effects of light in free space, Fig. 3. A related spin-Hall effect occurs upon focusing of light with a "half-lens" ( $\ell=0$ for $\delta=\pi / 2)^{26,28}$, whereas the orbital-Hall effect can also be observed upon focusing of asymmetric vortex beams. The values of $L_{z}$ and $S_{z}$ for the asymmetric beam are given by the same Eq. (12), but in this case the OAM has an extrinsic contribution, $L_{z}^{\mathrm{ext}}=-p_{x} y_{\ell}^{\sigma}(10)$ :

$$
L_{z}^{\mathrm{ext}}=\gamma^{2} L_{z}, \quad L_{z}^{\mathrm{int}}=\left(1-\gamma^{2}\right) L_{z} .
$$

This shows that the Hall effects of light can be interpreted as an intrinsic-to-extrinsic OAM conversion ${ }^{21,23,32}$ which is also accompanied by generation of a transverse OAM component $L_{x}^{\mathrm{ext}}=p_{z} y_{\ell}^{\sigma}=-\gamma \cot \theta_{0} L_{z}{ }^{29}$ The total conversion is achieved at $\delta \rightarrow 0, \gamma \rightarrow 1$.
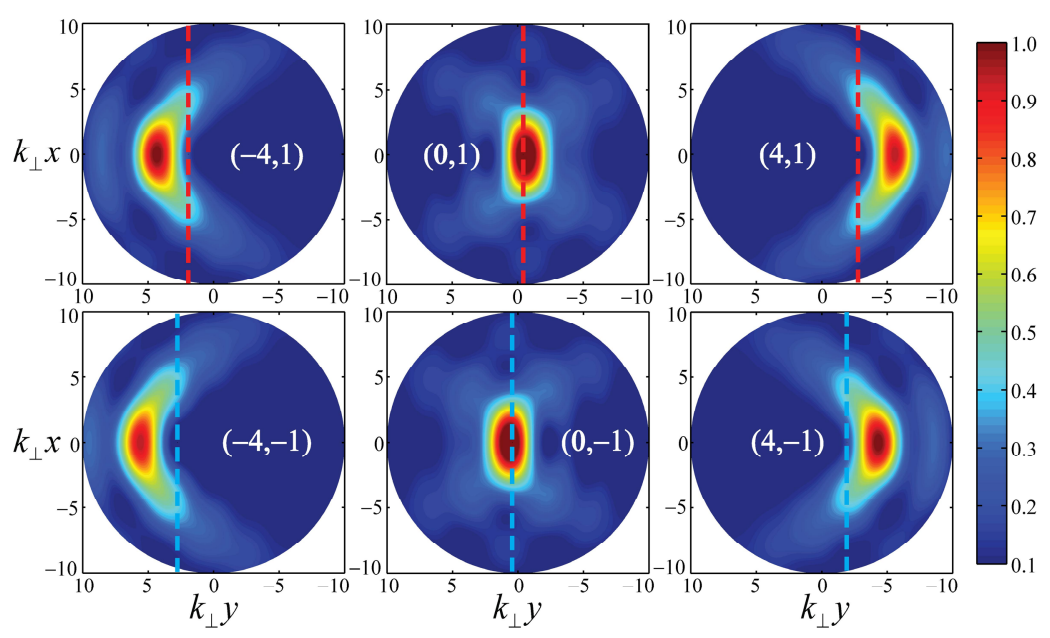

Figure 3. (Color online) Transverse intensity distributions at $z=0$ of the asymmetric Bessel beams with $\delta=\pi / 2$ and $\theta_{0}=3 \pi / 8$ marked by quantum numbers $(\ell, \sigma)$. Dashed lines indicate the $\ell$ - and $\sigma$-dependent transverse shifts of the centers of gravity, Eq. (15), i.e., orbital and spin Hall effects of light in free space. We have verified that the centers of gravity calculated numerically from the intensity distributions and theoretically from Eq. (15) coincide.

\section{CONCLUSION}

To summarize, we have given an exact self-consistent description of the OAM, SAM, position and SOI of nonparaxial light in free space. We have applied the general theory to symmetric and asymmetric Bessel-beam solutions which exhibit fine SOI splitting of caustics and Hall effects of light. These effects can be observed experimentally in tightly focused fields. 
This work was supported by the European Commission (Marie Curie fellowship), Science Foundation Ireland, and the Australian Research Council. We are indebted to A. Aiello, A. Bekshaev, and M. Berry for fruitful discussions.

\section{REFERENCES}

[1] Akhiezer, A. I. and Berestetskii, V. B., [Quantum electrodynamics] Interscience Publishers, New York (1965).

[2] van Enk, S. J. and Nienhuis, G., "Spin and orbital angular-momentum of photons," Europhys. Lett. 25, 497-501 (1994).

[3] van Enk, S. J. and Nienhuis, G., "Commutation rules and eigenvalues of spin and orbital angular momentum of radiation fields," J. Mod. Opt. 41, 963-977 (1994).

[4] Barnett, S. M. and Allen, L., "Orbital angular-momentum and nonparaxial light-beams," Opt. Commun. 110, 670-678 (1994).

[5] Barnett, S. M., "Optical angular-momentum flux," J. Opt. B: Quantum and Semiclass. Opt. 4, S7-S16 (2002).

[6] Allen, L., Beijersbergen, M. W., Spreeuw R. J. C. and Woerdman, J. P., "Orbital angular-momentum of light and the transformation of Laguerre-Gaussian laser modes," Phys. Rev. A 45, 8185-8189 (1992).

[7] Allen, L., Padgett, M. J. and Babiker, M., "The orbital angular momentum of light," Prog. Opt. 39, 291-372 (1999).

[8] Berry, M. V., "Optical currents," J. Opt. A: Pure Appl. Opt. 11, 094001 (2009).

[9] Li, C.-F., "Spin and orbital angular momentum of a class of nonparaxial light beams having a globally defined polarization," Phys. Rev. A 80, 063814 (2009).

[10] Bekshaev, A. Y., "A simple analytical model of the angular momentum transformation in strongly focused light beams," Cent. Eur. J. Phys. 8, 947-960 (2010).

[11] Pryce, M. H. L., "The mass-centre in the restricted theory of relativity and its connexion with the quantum theory of elementary particles," Proc. Roy. Soc. London, Ser. A 195, 62-81 (1948).

[12] Bialynicki-Birula, I. and Bialynicki-Birula, Z., "Berry phase in the relativistic theory of spinning particles," Phys. Rev. D 35, 2383-2387 (1987).

[13] Skagerstam, B.-S. K., "Localization of massless spinning particles and the Berry phase," arXiv:hep-th/9210054.

[14] Bérard, A. and Mohrbach, H., "Spin Hall effect and Berry phase of spinning particles," Phys. Lett. A 352, 190195 (2006).

[15] Bomzon, Z., Gu, M. and Shamir, J., "Angular momentum and geometrical phases in tight-focused circularly polarized plane waves," Appl. Phys. Lett. 89, 241104 (2006).

[16]Zhao, Y., Edgar, J. S., Jeffries, G. D. M., McGloin, D. and Chiu, D. T., "Spin-to-orbital angular momentum conversion in a strongly focused optical beam," Phys. Rev. Lett. 99, 073901 (2007).

[17] Schwartz, C. and Dogariu, A., "Conservation of angular momentum of light in single scattering," Opt. Express $14,8425-8433$ (2006).

[18] Haefner, D., Sukhov, S. and Dogariu, A., "Spin Hall Effect of light in spherical geometry," Phys. Rev. Lett. 102,123903 (2009).

[19] Rodríguez-Herrera, O. G., Lara, D., Bliokh, K. Y., Ostrovskaya, E. A. and Dainty, C., "Optical nanoprobing via spin-orbit interaction of light," Phys. Rev. Lett. 104, 253601 (2010).

[20] Liberman, V. S. and Zel'dovich, B. Y., "Spin-orbit interaction of a photon in an inhomogeneous-medium," Phys. Rev. A 46, 5199-5207 (1992).

[21] Onoda, M., Murakami, S. and Nagaosa, N., "Hall effect of light," Phys. Rev. Lett. 93, 083901 (2004).

[22] Bliokh, K. Y. and Bliokh, Y. P., "Topological spin transport of photons: the optical Magnus effect and Berry phase," Phys. Lett. A 333, 181-186 (2004).

[23] Bliokh, K. Y. and Bliokh, Y. P., "Conservation of angular momentum, transverse shift, and spin Hall effect in reflection and refraction of an electromagnetic wave packet," Phys. Rev. Lett. 96, 073903 (2006).

[24] Hosten, O. and Kwiat, P., "Observation of the spin Hall effect of light via weak measurements," Science 319, 787-790 (2008).

[25] Bliokh, K. Y., Niv, A., Kleiner, V. and Hasman, E., "Geometrodynamics of spinning light," Nature Photon. 2, 748-753 (2008).

[26] Baranova, N. B., Savchenko, A. Y. and Zel'dovich, B. Y., "Transverse shift of a focal spot due to switching of the sign of circular-polarization," JETP Lett. 59, 232-234 (1994). 
[27] Volyar, A. V. and Fadeeva, T. A., "Nonparaxial Gaussian beams: 2. Splitting of the singularity lines and the optical magnus effect," Tech. Phys. Lett. 26, 740-743 (2000).

[28] Bliokh, K. Y., Gorodetski, Y., Kleiner, V. and Hasman, E., "Coriolis effect in optics: Unified geometric phase and spin-Hall effect," Phys. Rev. Lett. 101, 030404 (2008).

[29] Aiello, A., Lindlein, N., Marquardt, C. and Leuchs, G., "Transverse angular momentum and geometric spin Hall effect of light," Phys. Rev. Lett. 103, 100401 (2009).

[30] Fedoseyev, V. G., "Spin-independent transverse shift of the centre of gravity of a reflected and of a refracted light beam," Opt. Commun. 193, 9-18 (2001).

[31] Dasgupta, R. and Gupta, P. K., "Experimental observation of spin-independent transverse shift of the centre of gravity of a reflected Laguerre-Gaussian light beam," Opt. Commun. 257, 91-96 (2006).

[32] Bliokh, K. Y., Shadrivov, I. V. and Kivshar, Y. S., "Goos-Hanchen and Imbert-Fedorov shifts of polarized vortex beams," Opt. Lett. 34, 389-391 (2009).

[33] Merano, M., Hermosa, N., Woerdman, J. P. and Aiello, A., "How orbital angular momentum affects beam shifts in optical reflection," Phys. Rev. A 82, 023817 (2010).

[34] Bliokh, K. Y., "Geometrical optics of beams with vortices: Berry phase and orbital angular momentum Hall effect," Phys. Rev. Lett. 97, 043901 (2006).

[35] Bliokh, K. Y. and Desyatnikov, A. S., "Spin and orbital Hall effects for diffracting optical beams in gradientindex media," Phys. Rev. A 79, 011807 (2009).

[36] Garbin, V., Volpe, G., Ferrari, E., Versluis, M., Cojoc, D. and Petrov, D., "Mie scattering distinguishes the topological charge of an optical vortex: a homage to Gustav Mie," New J. Phys. 11, 013046 (2009).

[37] Bekshaev, A. Y., "Oblique section of a paraxial light beam: criteria for azimuthal energy flow and orbital angular momentum," J. Opt. A: Pure Appl. Opt. 11, 094003 (2009).

[38] Hawton, M. and Baylis, W. E., "Photon position operators and localized bases," Phys. Rev. A 64, 012101 (2001).

[39] Hawton, M. and Baylis, W. E., "Angular momentum and the geometrical gauge of localized photon states," Phys. Rev. A 71, 033816 (2005).

[40] O'Neil, A. T., MacVicar, I., Allen, L. and Padgett, M. J., "Intrinsic and extrinsic nature of the orbital angular momentum of a light beam," Phys. Rev. Lett. 88, 053601 (2002).

[41] Aiello, A. and Bliokh, K. Y. (in preparation).

[42] As it is known, the Bessel beams cannot be normalized and we calculate the finite ratio of the quantities Eqs. (8)-(10) to the infinite norm $\left\langle\tilde{E}^{\sigma} \mid \tilde{E}^{\sigma}\right\rangle$.

[43] Shapere, A. and Wilczek, F., (ed) [Geometric Phases in Physics], World Scientific, Singapore (1989).

[44] Ngome, J.-P., Horváthy, P. A. and van Holten, J. W., "Dynamical supersymmetry of the spin particle-magnetic field interaction," J. Phys. A: Math. Theor. 43, 285401 (2010).

[45] Richards, B. and Wolf, E., "Electromagnetic diffraction in optical systems. 2. Structure of the image field in an aplanatic system," Proc. R. Soc. London A 253, 358-379 (1959).

[46] Berry, M. V. and McDonald, K. T., "Exact and geometrical optics energy trajectories in twisted beams," J. Opt. A: Pure Appl. Opt. 10, 035005 (2008).

[47] Mikitik, G. P. and Sharlai, Y. V., "Manifestation of Berry's phase in metal physics," Phys. Rev. Lett. 82, $2147-$ 2150 (1999).

[48] Gosselin, P., Boumrar, H. and Mohrbach, H., "Semiclassical quantization of electrons in magnetic fields: The generalized Peierls substitution," EPL 84, 50002 (2008).

[49] Novoselov, K. S., Geim, A. K., Morozov, S. V., Jiang, D., Katsnelson, M. I., Grigorieva, I. V., Dubonos, S. V. and Firsov, A. A., "Two-dimensional gas of massless Dirac fermions in grapheme," Nature 438, 197-200 (2005).

[50]Zhang, Y. B., Tan Y. W., Stormer, H. L. and Kim, P., "Experimental observation of the quantum Hall effect and Berry's phase in grapheme," Nature 438, 201-204 (2005).

[51] Bomzon, Z. and Gu, M., "Space-variant geometrical phases in focused cylindrical light beams," Opt. Lett. 32, 3017-3019 (2007).

[52] Gorodetski, Y., Niv., A., Kleiner, V. and Hasman, E., "Observation of the spin-based plasmonic effect in nanoscale structures" Phys. Rev. Lett. 101, 043903 (2008). 